\title{
Assessment of Effective Risk in Software Projects based on Wallace's Classification Using Fuzzy Logic
}

\author{
Ali Yavari \\ Mazandaran University of Sciences and Technology, Iran \\ Email:yavari@ustmb.ac.ir \\ Maede Golbaghi \\ Mazandaran University of Sciences and Technology, Iran \\ Email: maede.golbaghi@gmail.com \\ Hossein Momeni \\ Agricultural Sciences and Natural Resources University of Gorgan, Iran \\ Email: momeni@gau.ac.ir
}

\begin{abstract}
Software development always faces unexpected events such as technology changes, environmental changes, changing user needs. These changes will increase the risk in software projects. We need to risk management to deal with software risks. Risk assessment is one of the most important factors in risk and project management of software projects. In this paper, we use Wallace's work and five factors to present an efficient method to measure software risk using fuzzy logic. Team, Planning, Complexity, Requirements and User are factors that we use in this paper. Results of experiments shows that our framework is more efficient than other frameworks and approaches for risk assessment in software projects.
\end{abstract}

Index Terms - Software Risk, Assessment, Wallace's Classification, Fuzzy Logic.

\section{INTRODUCTION}

Software development projects face a number of software risks. The most important factors that may cause failure of project are related to poor performance, team pressure, low quality and high cost [1]. So software project risk management plays an important role in completing software projects successfully. It consists of the following four phases: identification, assessment, plan and control. Risk assessment is the base of software project risk management. According to [2] definition, risk exposure is expressed as the relationship $\mathrm{RE}=\mathrm{P}(\mathrm{uo}) * \mathrm{~L}(\mathrm{uo})$, where $\mathrm{RE}$ is the risk exposure, $\mathrm{P}(\mathrm{uo})$ is the probability of an unsatisfactory outcome and L(uo) is the loss to the parties affected if the outcome is unsatisfied[3].

The major problem associated with the estimation of risks is that the input data are imp recise by nature and it is difficult to represent them with crisp numbers. Usually the risk analyst prefers to estimate in linguistic terms such as high or low rather than in exact probabilistic terminology. To this end, the application of Fuzzy Set Theory (FST) to risk analysis seems appropriate; as such analysis can handle subjectivity as well as inexact and vague information [1].

The solution that is suggested here to overcome previously mentioned problems is to use fuzzy logic linguistic variables for the complexity metrics and model. Fuzzy logic is a mathematical tool for dealing with uncertainties and also it provides a technique to deal with imprecision and information granularity. Fuzzy logic is seen as a means of approximate reasoning. The fuzzy logic has been successfully applied in many segments such as engineering, psychology, artificial intelligence, medicine and sociology. In this paper we propose a fuzzy logic approach for risk estimation in software projects. We use five factors that are important in risk management. Those factors are Team, Planning, Complexity, Requirements and User.

Our fuzzy model has five input and we use 3 linguistic variables for each input. This membership functions are Low, Medium and High. Fuzzification module maps the non-fuzzy values in fuzzy space and defuzzification module convert the fuzzy numbers into crisp space. In our framework, after fuzzificaition of each input, the Mamdani inference system that includes a rule base can evaluate the risk of software project. This is a fuzzy number, so it should convert to a crisp number by a defuzzificaion algorithm. Number of rules in this rule base that use in this system is 243 and "And Method " is min also "Or Method" is max, th is mean that and operator between two numbers select minimum of numbers and or operator between two number select maximum of this numbers. Fuzzy inference is the actual process of mapping from a given input to an output using fuzzy logic. The process involves all the pieces: membership functions, fuzzy logic operators, and ifthen rules. Results of experiments shows that our framework is more efficient than other frameworks and approaches for risk assessment in software projects. 
The rest of this paper is organized as follows: Section 2 describes the related works. Section 3 describes the concepts and definitions. Section 4 explains the Wallace's Work. Section 5 describes proposed approach and finally section 6 concludes the paper with some discussion.

\section{RELATED WORK}

This section presents works that related to risk and risk management. Boehm [2] proposed a software risk management framework. He identified a list of the topten software risks based on his experience at TRW. There were some problems in his survey. The list of topten software risks lacked a theoretical foundation. Secondly, these risks are set according to software development environment in 1991 but scale and diversity of software have increased and thus, the list has become inadequate.

Barki et al. [4] conducted a survey in Quebec. They identified a list which included 23 software risks. They classified them into five groups. The list provided a comprehensible instrument but Wallace et al.[13] explained that the assessment scale of each risk was complex.

Schmidt et al. conducted a Delphi survey to reduce the bias of a single-culture viewpoint. They integrated the options of many experts from some countries. They identified 53 risk items and grouped them into 14 types. They declared that cultural difference could affect the list. Only 11 software risks were applicable from a cross-cultural perspective [14]. Recently, Wallace et al. collected the opinions of 507 members in the Project Management Institute (PMI) and identified 27 software risks, which were classified into six dimensions: User, Requirement, Project Complexity, Planning \& Control, Team and Organizational Environment using structural Equation Model. A summary of related studies on software risks is given in table 1[4]. Th is table has seven rows, Project Type, Scope, Participant, Participant numbers, Research Method, Dimensions, Dimensions and finally risk.
TABLE 1: summary of related studies on soft ware risks

\begin{tabular}{|c|c|c|c|}
\hline Project Type & General & General & General \\
\hline Scope & TRW & Quebec & $\begin{array}{c}\text { Across } \\
\text { Countries }\end{array}$ \\
\hline Participant & $\begin{array}{c}\text { Project } \\
\text { Manager }\end{array}$ & $\begin{array}{c}\text { Project Leader } \\
\text { and User } \\
\text { representative }\end{array}$ & $\begin{array}{c}\text { PMI } \\
\text { members }\end{array}$ \\
\hline $\begin{array}{c}\text { Participant } \\
\text { numbers }\end{array}$ & Unknown & 120 & 507 \\
\hline $\begin{array}{c}\text { Research } \\
\text { Method }\end{array}$ & Unknown & CFA & SEM \\
\hline Dimensions & 0 & 5 & 6 \\
\hline Risks & 10 & 23 & 27 \\
\hline
\end{tabular}

In our study, five of six risk dimensions of Wallace's work were adopted. Firstly, her work was conducted in 2004, and thus it was relatively up-to-date and reflected the consensus of 507 PMI members from various countries. Secondly, SEM was used in her work to examine and prove the composite reliability, convergent validity and adequacy of the proposed framework of software risks. Therefore, the six risk dimensions and their associated software risks, as shown in table 2, were considered appropriate for our study.

This table has two columns, factors and sub factors. Factors are: user, requirement, project complexity, planning and control, team and organizational environment. For example sub factors of planning and control are: lack of an effective project management methodology, project progress not monitored closely enough, inadequate estimation of required resources, poor project planning, project milestones not clearly defined, inexperienced project manager and ineffective communication. Also sub factors of user are: Users resistant to change, Conflict between users, Users with negative attitudes toward the project, Users not committed to the project and Lack of cooperation from users. 
TABLE 2: Software risks adopted in this study

\begin{tabular}{|c|c|}
\hline Risk factor & Sub factors of risk \\
\hline User & $\begin{array}{ll}\text { - } & \text { Users resistant to change } \\
\text { - } & \text { Conflict bet ween users } \\
\text { - } & \text { Users with negat ive att itudes } \\
\text { - } & \text { Users nord the project } \\
\text { - } & \text { Lroject } \\
\text { Lack of cooperation from users }\end{array}$ \\
\hline Requirement & $\begin{array}{ll}- & \text { Continually changing system } \\
\text { - } & \text { requirements } \\
& \text { System requirements not } \\
\text { - } & \text { Udequately identified } \\
\text { - } & \text { Inclear system requirements } \\
& \text { Incorrect system requirements }\end{array}$ \\
\hline Project complexity & $\begin{array}{l}\text { - } \\
\text { Project involved the use of new } \\
\text { technology } \\
\text { High level of technical } \\
\text { complexity } \\
\text { - } \quad \text { Immature technology } \\
\text { Project involves the use of } \\
\text { technology that has not been used } \\
\text { in prior projects }\end{array}$ \\
\hline $\begin{array}{l}\text { Planning and } \\
\text { control }\end{array}$ & $\begin{array}{ll}- & \text { Lack of an effective project } \\
\text { management methodology } \\
\text { - } & \text { Project progress not monitored } \\
\text { closely enough } \\
\text { - } & \text { Inadequate estimation of required } \\
\text { - } & \text { resources } \\
\text { - } & \text { Poor project planning } \\
\text { - } & \text { defined milestones not clearly } \\
\text { - } & \text { Inexperienced project manager } \\
& \text { Ine communication }\end{array}$ \\
\hline Team & $\begin{array}{ll}\bullet & \text { Inexperienced team members } \\
\text { - } & \text { Inadequat ely trained development } \\
\text { team members } \\
\text { - } \quad \text { Team members lack specialized } \\
\text { skills required by the project }\end{array}$ \\
\hline $\begin{array}{l}\text { Organizational } \\
\text { environment }\end{array}$ & $\begin{array}{l}\text { - Change in organizational } \\
\text { management during the project } \\
\text { - } \quad \text { Corporate politics with negative } \\
\text { effect on the project } \\
\text { - Unstable organizational } \\
\text { environment } \\
\text { - Organization undergoing } \\
\text { restructuring during the project }\end{array}$ \\
\hline
\end{tabular}

\section{CONCEPTS AND DEFINITIONS}

In this section we have review on some concepts and definition.

\subsection{Risk}

Risk arises when organizations pursue opportunities in the face of uncertainty, constrained by capability and cost. The most common definition of risk in software projects is in terms of exposure to specific factors that present a threat to achieving the expected outcomes of a project [7]. A software risk (an uncertain event or condition with negative consequences on a software project) can increase the failure rate of a project if it is ignored [8]. Thus, the main purpose of software risk management is to identify managerial and technical problems before they occur so that actions can be taken to eliminate or mitigate their impact [6].
Currently, measuring the grade of risk mainly depends on the value of risk exposure. According to Boehm's definition, risk exposure is expressed as the relationship $\mathrm{RE}=\mathrm{P}(\mathrm{uo}) * \mathrm{~L}(\mathrm{uo})$, where $\mathrm{RE}$ is the risk exposure, $\mathrm{P}(\mathrm{uo})$ is the probability of an unsatisfactory outcome and L(uo) is the loss to the parties affected if the outcome is Unsatisfied[3].

Risk in software projects is usually defined as the probability-weighted impact of an event on a project. In classical decision theory, risk was viewed as reflecting variation in the probability distribution of possible outcomes, negative or positive, associated with a particular decision [7].

There are two classes of software project risk: generic risks common to all projects, and project-specific risks. Some of these risks are easy to identify and manage. Others are less obvious or it is more difficult to predict their likelihood and/or impact. This is complicated by multiple project dimensions including size, structure, complexity, composition, context, novelty, long planning and execution horizons, and volatile change. Therefore, risk management in software projects is important to: help avoid disasters; avoid rework; focus and balance effort; and stimulate win-win situations [7].

\subsection{Risk Management}

As foreshadowed above, software project risk management is usually defined as a set of principles and practices aimed at identifying, analyzing and handling risk factors to improve the chances of achieving a successful project outcome and/or avoid project failure. Any variation in approach is usually in the 'principles and practices' employed within this conceptual understanding of risk management.

Most commonly, one or more of four inter-related approaches to risk management are found in the literature and practice. These are checklists, analytical frameworks, process models, and risk response strategies [7].

Risk management can lead to a range of project and organizational benefits including:

- Identification of favourable alternative courses of action;

- Increased confidence in achieving project objectives;

- $\quad$ imp roved chances of success;

- $\quad$ Reduced surprises;

- More precise estimates (through reduced uncertainty);

- $\quad$ Reduced duplication of effort (through team awareness of risk control actions) [7].

\section{WALLACE'S WORK}

In our study, the six risk dimensions of Wallace's work were adopted. Firstly, her work was conducted in 2004, and thus it was relatively up-to-date and reflected the consensus of 507 PMI members from various 
countries. Secondly, SEM was used in her work to examine and prove the composite reliability, convergent validity and adequacy of the proposed framework of software risks. In her work 27 software risks, which were classified into six dimensions: User, Requirement, Project Complexity, Planning \& Control, Team and Organizational Environment using structural Equation Model [6].

\section{PROPOSED APPROACH}

Before presenting the proposed method, let's review some basic definitions related to fuzzy logic.

\section{Definiti on 1:}

If our universe is $\mathrm{X}$, then fuzzy set $\mathrm{A}$ would be as follows:

$$
A=\left\{<x, \mu_{A}(x)>: x \in X\right\}
$$

Membership function $\mu_{\mathrm{A}}(\mathrm{x})$ when $\mathrm{x}$ is a member and $\mu_{A}: X \rightarrow[0,1]$ : specifies degree of membership $x$ to set A $[8,9]$

\section{Definition 2:}

$$
A=\left\{<x, \mu_{A}(x), V_{A(x)}>\mid x \in X\right\}
$$

Here $\mu_{\mathrm{A}}: \mathrm{X} \rightarrow[0,1]$ and $\mathrm{V}_{\mathrm{A}}: \mathrm{X} \rightarrow[0,1]$ with the following condition $0 \leq \mu_{\mathrm{A}}(\mathrm{x})+\mathrm{V}_{\mathrm{A}}(\mathrm{x}) \leq 1, \forall \mathrm{x} \in$ $\mathrm{X}$

$\mu_{\mathrm{A}}(\mathrm{x})$ Specifies degree of membership and $V_{\mathrm{A}}(\mathrm{x})$ specifies degree of non-membership.in this example we use triangular membership function and explain it below $[9,10]$.

\section{Definiti on 3:}

A triangular fuzzy number can be sorted with a trio (1, $\mathrm{m}, \mathrm{u}$ ) that 1 and $\mathrm{u}$ are upper and lower limits, $\mathrm{m}$ is middle and $\mathrm{x}$ is an element between 1 and 0 as shown in Figure 1.

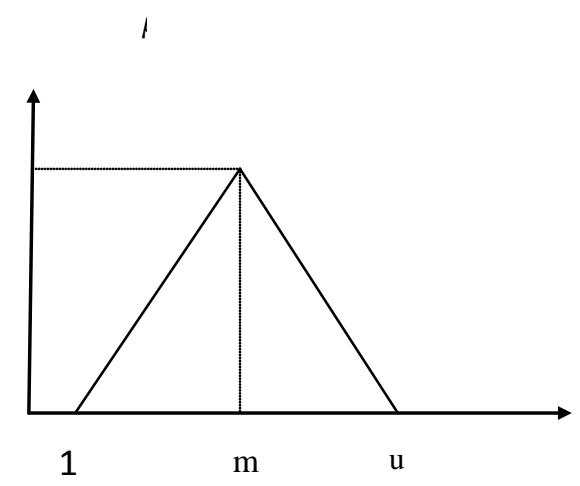

Figure 1:Triangular fuzzy number
And also:

$\mu(x)$
$= \begin{cases}\frac{x-1}{m-1}, & 1 \leq x<m ; \\ \frac{u-x}{u-m} \mu_{\tilde{a}}, & m<x<u\end{cases}$

For defuzzification of risk final amount we can use center of gravity technique. This technique developed by Sugeno in 1985 . This technique is most commonly used method and is very accurate. Center of gravity technique is described as follows [11-13].

$x^{*}=\frac{\int \mu_{i}(x) x d x}{\int \mu_{i} x d x}$

$\mathrm{x}^{*}$ Defuzzy output, $\mathrm{x}^{*}$ total membership function and $\mathrm{x}$ is output variable.

In this paper we use five factors that described in Wallace's work. These factors are user, requirements, complexity, planning and Team. Figure 1 shows the model. In this model, the final output of the projects software risks are due to five factors.

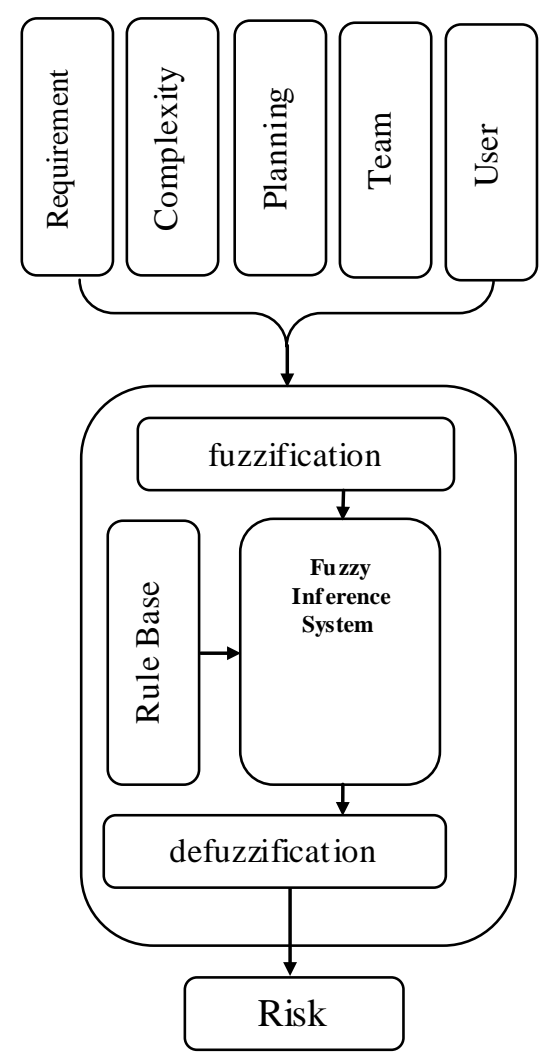

Figure 2: Software risk assessment model 
TABLE 3: Implementation of fuzzy inference system

\begin{tabular}{|c|c|}
\hline System & $\begin{array}{c}\text { Name='RiskEvaluation', Type='mamdani', Version=2.0, NumInputs=5, NumOutputs=1, } \\
\text { NumRules=243, AndMethod='min', OrMethod='max', ImpMethod='min', } \\
\text { AggMethod='max', DefuzzMethod='centroid' }\end{array}$ \\
\hline Input1 & 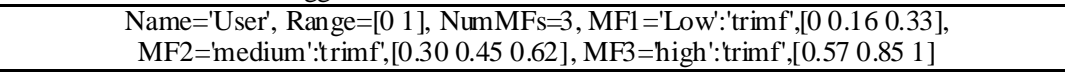 \\
\hline Input2 & 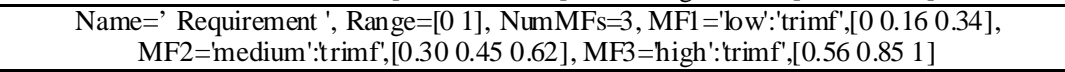 \\
\hline Input3 & 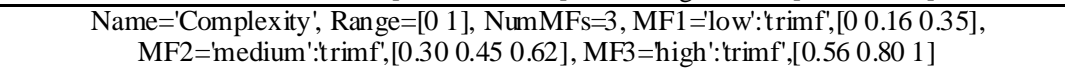 \\
\hline Input4 & 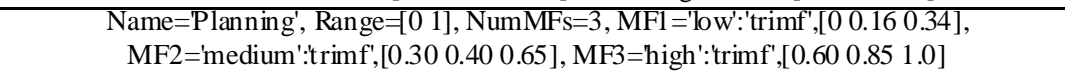 \\
\hline Input5 & 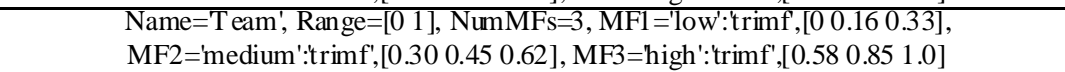 \\
\hline Output & 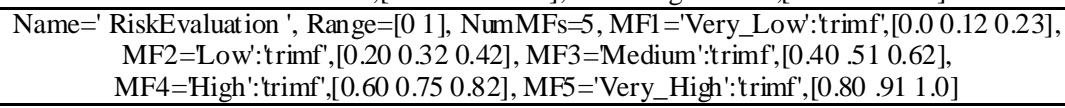 \\
\hline
\end{tabular}

Output categorize in five category that are very low risk project ( Very Low ), Low-risk project ( Low ), Medium-risk project ( Medium ), High-risk project ( High ) and very high risk Project ( Very High ). We use 3 linguistic variables for each input. Number of rules generated is equal to 243. We can see some of generated rules below. According to input data for each input, one of the rules is activated.

Number of generated rules is equal to 243. Corresponding Membership functions cut and after defuzzification, final output calculates.

\subsection{Rules description}

We have five input and we use 3 linguistic variables for each input. Therefore numbers of rules are 243 . The following block of code shows some rules:

Rule 1: If (User is Low) and (Requirement is Low) and (Complexity is Low) and (Planning is

Low) and (team is Low) then (Risk is Very Low)

Rule 2: If (User is Low) and (Requirement is Low) and (Complexity is Low) and (Planning is Low) and (team is Medium) then (Risk is Low)

Rule3: If (User is Low) and (Requirement is Low) and (Complexity is Low) and (Planning is Low) and (team is High) then (Risk is Low)

Rule4: If (User is Low) and (Requirement is Low) and (Complexity is Low) and (Planning is Medium) and (team is High) then (Risk is Low)

Rule5: If (User is Low) and (Requirement is Low) and (Complexity is Low) and (Planning is High) and (team is High) then (Risk is Low)

Rule6: If (User is Low) and (Requirement is Low) and (Complexity is Medium) and (Planning is Low) and (team is High) then (Risk is Low)

Rule7: If (User is Low) and (Requirement is Low) and (Complexity is High) and (Planning is Low) and (team is High) then (Risk is Low)

Rule8: If (User is Low) and (Requirement is Medium) and (Complexity is Low) and (Planning is Low) and (team is High) then (Risk is Low)
Rule9: If (User is Low) and (Requirement is High) and (Complexity is Low) and (Planning is Low) and (team is High) then (Risk is Low)

Rule241: If (User is High) and (Requirement is High) and (Complexity is High) and (Planning is High) and (Team is Low) then (Risk is High)

Rule242: If (User is High) and (Requirement is High) and (Complexity is High) and ( Planning is High) and (Team is Medium) then (Risk is Very High )

Rule243: If (User is High) and (Requirement is High) and (Complexity is High) and (Planning is High) and (Team is High) then (Risk is Very High)

Mamdani inference used to implement the fuzzy inference system. System information is presented in Table 3. According to table 3 this system has 5 inputs and 1 output. Inputs are: User Requirement, Project complexity, Planning and Team. Number of fuzzy rules that use in this system is 243 and "AndMethod " is min also "OrMethod" is max, this mean that and operator between two numbers select minimum of numbers and or operator between two number select maximum of this numbers. Range of each inputs, output and fuzzy linguistic variable is shown in table 3 . For example: input 2 has 3 membership function named: Low, Medium and High. Range of Low is $0.0-0.33$, Medium is $0.30-0.62$ and High is $0.57-1.0$ and type of membership function is trimf. Also for output variable that is value of software project risk, there are five membership function that name and range of them are: Very Low [ $\left.\begin{array}{lll}0.0 & 0.12 & 0.23\end{array}\right]$, Low [ $\left.\begin{array}{lll}0.20 & 0.32 & 0.42\end{array}\right]$, Medium [0.40 .51 0.62], High [0.60 0.75 0.82] and Very High [0.80 .91 1.0], type of those membership functions are trimf and name of this output variable is risk evaluation in Mamdani inference.

\subsection{Membership Function Assignment}

The following example illustrates how the membership grade is assigned to output. The inputs are fed to the fuzzification module and after fuzzification of given values we find that user $=0.90$ belongs to High membership function, requirement $=0.87$ belongs to 
High membership function, complexity $=0.64$ belongs to High membership function, planning $=0.76$ belongs to High membership function and team $=0.18$ belongs to Low membership function, so:

Rule\#: if (user=0.90) and (requirement $=0.87$ ) and (complexity $=0.64)$ and (planning=0.76) and (team=0.18) then (?)

With these input values the following rule gets fired:

Rule241: If (User is High) and (Requirement is High) and (Complexity is High) and (Planning is High) and (Team is Low) then (Risk is High)

The rule gives the output value as High which indicates the high risk for software project.

Another example of how the membership grade is assigned to output:

User $=0.11$ belongs to Low membership function, requirement $=0.18$ belongs to Low membership function, complexity $=0.31$ belongs to Low membership function, planning $=0.06$ belongs to Low membership function and team $=0.25$ belongs to Low membership function, so:

Rule\#: if (user=0.11) and (requirement $=0.18$ ) and $($ complexity $=0.31)$ and (planning $=0.06)$ and (team=0.25) then (?)

With these input values the following rule gets fired.

Rule1: If (User is Low) and (Requirement is Low) and (Complexity is Low) and (Planning is Low) and (Team is Low) then (Risk is Very Low)

The rule gives the output value as Very Low which indicates the Very Low risk for software project.

\subsection{Assessment}

The proposed model was implemented for four projects. Details of these projects are described in table 4.

Table 4: System results for some projects

\begin{tabular}{|c|c|c|c|c|}
\hline & $\begin{array}{c}\text { Project } \\
1\end{array}$ & $\begin{array}{c}\text { Project } \\
2\end{array}$ & $\begin{array}{c}\text { Project } \\
3\end{array}$ & $\begin{array}{c}\text { Project } \\
4\end{array}$ \\
\hline Team & 0.198 & 0.752 & 0.988 & 0.380 \\
\hline Planning & 0.134 & 0.907 & 0.875 & 0.537 \\
\hline Complexity & 0.089 & 0.085 & 0.798 & 0.434 \\
\hline Requirement & 0.165 & 0.077 & 0.909 & 0.614 \\
\hline User & 0.251 & 0.124 & 0.034 & 0.555 \\
\hline $\begin{array}{c}\text { Risk } \\
\text { Evaluation }\end{array}$ & $\mathbf{0 . 1 8 2}$ & $\mathbf{0 . 7 9 8}$ & $\mathbf{0 . 8 1 9}$ & $\mathbf{0 . 5 9 2}$ \\
\hline
\end{tabular}

For example in project $\mathrm{p} 1$, after fuzzification of inputs, user factor equals 0.251 , requirement factor equal to 0.165 , the complexity factor equals 0.089 , the planning factor is equal to 0.134 and team factor is equal to 0.198 . According to these characteristics, rule 1 gets fired and by defuzzification of fuzzy value with center of gravity method, output was equaled to 0.182 . Another example of this table is p3, after fuzzification of inputs, user factor equals 0.034 , requirement factor equal to 0.909 , the complexity factor equals 0.798 , the planning factor is equal to 0.875 , and team factor is equal to 0.988 .

After firing the rule and defuzzification of fuzzy value with center of gravity method, output was equaled to 0.819

\section{CONCLUSION}

In this paper we made short review of risk management, classification of it and factors that affecting it and presented an efficient method to measure software risk using fuzzy logic. This fuzzy system has 5 inputs and one output. Number of fuzzy rules in this system is 243 and each input has 3 membership functions. The presented system is based on 5 factors: Team, Planning, Complexity, Requirements and User. Those factors are very important for managers of software projects. Very important decisions in software projects are made on these factors. The presented system was tested on four projects and risk of the projects was calculated. Software project team can use this information to make appropriate decisions in order to deal with the project.

\section{ACKNOW LEDGMENTS}

This research was partially supported by the Agricultural Sciences and Natural Resources University of Gorgan and Mazandaran University of Sciences and Technology.

\section{REFERENCES}

[1] Lazzerini, Beatrice, and Lusine Mkrtchyan. "Analyzing risk impact factors using extended fuzzy cognitive maps." Systems Journal, IEEE 5.2 (2011): 288-297.

[2] Boehm, Barry W. "Software risk management: principles and practices." Software, IEEE 8.1 (1991): 32-41.

[3] Li, Yang, and Nan Li. "Software project risk assessment based on fuzzy linguistic multiple attribute decision making." Grey Systems and Intelligent Services, 2009. GSIS 2009. IEEE International Conference on. IEEE, 2009.

[4] H. Barki, S. Rivard, J. Talbot, Toward an assessment of software development risk, Journal of Management Information Systems 10 (2), 1993, pp. 203-225.

[5] R. Schmidt, et al., Identifying software project risks: an international Delphi study, Journal of Management Information Systems 17 (4), 2001, pp. 5-36. 
[6] Huang, Sun-Jen, and Wen-Ming Han. "Exploring the relationship between software project duration and risk exposure: A cluster analysis." Information \& Management 45.3 (2008): 175-182

[7] Bannerman, Paul L. "Risk and risk management in software projects: A reassessment." Journal of Systems and Software 81.12 (2008): 2118-2133.

[8] T. DeMarco, T. Lister, Waltzing With Bears: Managing Risk on Software Projects, Dorset House Publishing Company, 2003.

[9] Atanassov, Krassimir T. "Intuitionistic fuzzy sets." Fuzzy sets and Systems 20.1 (1986): 87-96.

[10] Grzegorzewski, Przemysław. "Distances between intuitionistic fuzzy sets and/or interval-valued fuzzy sets based on the Hausdorff metric." Fuzzy Sets and Systems 148.2 (2004): 319-328.

[11] Wang, Zhoujing, Linfeng Wang, and Kevin W. Li. "A linear programming method for interval-valued intuitionistic fuzzy multiattribute group decision making." Control and Decision Conference (CCDC), 2011 Chinese. IEEE, 2011.

[12] Wei, Guiwu. "Some arithmetic aggregation operators with intuitionistic trapezoidal fuzzy numbers and their application to group decision making." Journal of Computers 5.3 (2010): 345351.

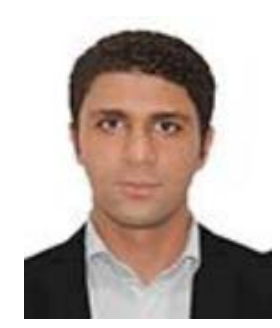

Ali Yavari received his Master of Science degree in Information Technology from Mazandaran University of Science and Technology (MUST) and works on Software Complexity in agentoriented Software development. His research interests include fuzzy sets and systems, neural networks, fuzzy neural networks, clustering and classification algorithms in data mining, software risk and also complexity in aspect and agent-oriented methodologies.

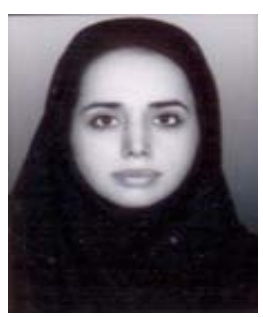

Maedeh Golbaghi received her M.Sc. in Information Technology Engineering form Mazandaran University of Science and Technology (MUST), Mazandaran, Iran. Her research interests are in the areas of optimization, software engineering, data preprocessing, risk management and fuzzy logic and data mining.

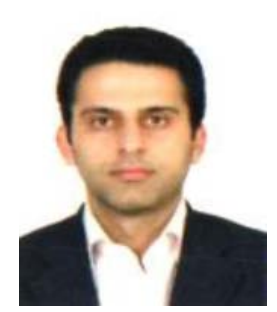

Hossein Momeni is an Assistant Professor of Software Engineering in Agricultural Sciences and Natural Resources University of Gorgan. He received the B.Sc. degree in software engineering from The Ferdowsi University of Mashhad, Mashhad, Iran and the M.Sc. and Ph.D. in software engineering from Iran University of Science and Technology (IUST), Tehran, Iran. His research interests include distributed system, real-time scheduling, wireless sensor actor networks and software engineering. 